\title{
COMMUTATORS OF BMO FUNCTIONS AND SINGULAR INTEGRAL OPERATORS WITH NON-SMOOTH KERNELS
}

\author{
Xuan Thinh Duong and Lixin Yan
}

Let $\mathcal{X}$ be a space of homogeneous type of infinite measure. Let $T$ be a singular integral operator which is bounded on $L^{p}(\mathcal{X})$ for some $p, 1<p<\infty$. We give a sufficient condition on the kernel of $T$ so that when a function $b \in B M O(\mathcal{X})$, the commutator $[b, T](f)=T(b f)-b T(f)$ is bounded on $L^{p}$ spaces for all $p, 1<p<\infty$. Our condition is weaker than the usual Hörmander condition. Applications include $L^{p}$-boundedness of the commutators of BMO functions and holomorphic functional calculi of Schrödinger operators, and divergence form operators on irregular domains.

\section{INTRODUCTION}

Let $(\mathcal{X}, d, \mu)$ be a space of homogeneous type, equipped with a metric $d$ and a measure $\mu$. Let $T$ be a bounded operator on $L^{p}(\mathcal{X})$ for some $p, 1<p<\infty$. A measurable function $K(x, y)$ is called an associated kernel of $T$ if

$$
T(f)(x)=\int_{\mathcal{X}} K(x, y) f(y) d \mu(y)
$$

holds for each continuous function $f$ with compact support, and for almost all $x$ not in the support of $f$.

The kernel $K(x, y)$ is said to satisfy

(i) the pointwise Hörmander condition on $x$ variable if there exist $0<\alpha \leqslant 1$ and $c_{1} \geqslant 1$ such that

$$
|K(x, y)-K(z, y)| \leqslant c \frac{1}{\mu(B(x ; d(x, y))} \frac{d(x, z)^{\alpha}}{d(x, y)^{\alpha}},
$$

when $d(x, y) \geqslant c_{1} d(x, z)$, and $B(x ; r)$ denotes the ball with centre $x$, radius $r$

\section{Received 30th May, 2002}

Both authors are partially supported by a grant from Australia Research Council. The second author is also partially supported by NSF of China. The authors thank J.M. Martell for making [11] available to us.

Copyright Clearance Centre, Inc. Serial-fee code: 0004-9727/03 \$A2.00+0.00. 
(ii) the integral Hörmander condition on $y$ variable if there exist constants $C$ and $c_{2} \geqslant 1$ such that

$$
\int_{d(x, y) \geqslant c_{2} d(z, y)}|K(x, y)-K(x, z)| d \mu(x) \leqslant C,
$$

for all $y, z \in \mathcal{X}$.

It is well-known that if $T$ is bounded on $L^{q}(\mathcal{X})$ for some $q, 1<q<\infty$, and $b \in B M O$, the two Hörmander conditions (i) and (ii) above are sufficient to imply that the commutator $[b, T] f$ is bounded on $L^{p}(\mathcal{X})$ for all $p, 1<p<\infty$, with norm

$$
\|[b, T](f)\|_{p} \leqslant C\|b\|_{*}\|f\|_{p},
$$

where the commutator $[b, T]$ is defined by $[b, T](f)=b T f-T(b f)$ and $\|b\|_{*}$ is the BMO semi-norm of $b$. See $[5, \mathbf{1 0}]$ for BMO functions on Euclidean spaces $\mathcal{X}=\mathbb{R}^{n}$, and [3] for spaces of homogeneous type.

Our aim of this paper is to replace the two Hörmander conditions (1.2) and (1.3) by the following weaker conditions (1.4) and (1.5) below which previously appeared in [8], and still conclude that the commutator $[b, T]$ is bounded on $L^{p}(\mathcal{X})$ for all $p, 1<p<\infty$. Roughly speaking, for the case $\mathcal{X}$ has infinite measure, we assume the following.

(iii) There exists a class of operators $A_{t}$ with kernels $a_{t}(x, y)$, which satisfy the condition (2.3) in Section 2, so that the kernels $k_{t}(x, y)$ of the operators $\left(T-A_{t} T\right)$ satisfy the condition

$$
\left|k_{t}(x, y)\right| \leqslant c_{1} \frac{1}{\mu(B(x ; d(x, y))} \frac{t^{\alpha / m}}{d(x, y)^{\alpha}}
$$

when $d(x, y) \geqslant c_{2} t^{1 / m}$ for some $\alpha, m>0$; and

(iv) There exists a class of operators $\left\{B_{t}, t>0\right\}$ satisfying condition (2.3) such that $\left(T-T B_{t}\right)$ have associated kernels $K_{t}(x, y)$ and there exist positive constants $c_{3}, c_{4}$ such that

$$
\int_{d(x, y)>c_{3} t^{1 / m}}\left|K_{t}(x, y)\right| d \mu(x) \leqslant c_{4}, \quad \text { for all } y \in \mathcal{X} .
$$

Under conditions (1.4) and (1.5), if $T$ is bounded on $L^{q}(\mathcal{X})$ for some $q, 1<q<\infty$, then the commutator $[b, T] f$ is bounded on $L^{p}(\mathcal{X})$ for all $p, 1<p<\infty$.

Note that the classes of operators $A_{t}$ and $B_{t}$ play the role of generalised approximations to the identity. It is not difficult to check that conditions (1.4) and (1.5) are consequences of conditions (1.2) and (1.3), respectively. See, [8, Proposition 2].

The paper is organised as follows. In Section 2 we give definitions of space of homogeneous type and the sharp maximal function $M_{A}^{\#} f$ associated with a "generalised 
approximations to the identity" $\left\{A_{t}, t>0\right\}$ as in [11]. We also recall some known results and prove a lemma there. Our main result is Theorem 3.1 in Section 3. Our proof does not use the usual Fefferman-Stein sharp maximal function $f^{\#}$. Instead, we use the sharp maximal function $M_{A}^{\#} f$ which was previously introduced in [11]. In Section 4, we extend our result to the case of the underlying space being a subset of a space of homogeneous type of infinite measure. In the last section, applications are given to holomorphic functional calculi of Schrödinger operators, and divergence form operators on irregular domains.

We note that there is difference in estimates for boundedness of commutators of BMO functions and singular integral operators when the underlying space $\mathcal{X}$ has finite measure and when $\mathcal{X}$ has infinite measure. In this paper, we only study the case $\mu(\mathcal{X})=\infty$. The case $\mu(\mathcal{X})<\infty$ will be studied in a seperate paper [9].

Throughout, the letter " $C$ " will denote (possibly different) constants that are independent of the essential variables.

\section{DEFINITIONS AND PRELIMINARY RESULTS}

Let $\mathcal{X}$ be a topological space equipped with a measure $\mu$ and a metric $d$ which is a measurable function on $\mathcal{X} \times \mathcal{X}$. We define $\mathcal{X}$ to be a space of homogeneous type if the balls $B(x ; r)=\{y \in \mathcal{X}: d(y, x)<r\}$ satisfy the doubling property

$$
\mu(B(x ; 2 r)) \leqslant c \mu(B(x ; r))<\infty
$$

for some $c \geqslant 1$ uniformly for all $x \in \mathcal{X}$ and $r>0$. A more general defintion can be found in [6, Chapter 3].

Note that the doubling property implies the following strong homogeneity property,

$$
\mu(B(x ; \lambda r)) \leqslant c \lambda^{n} \mu(B(x ; r))
$$

for some $c, n>0$ uniformly for all $\lambda \geqslant 1$. The parameter $n$ is a measure of the dimension of the space. There also exist $c$ and $N, 0 \leqslant N \leqslant n$ so that

$$
\mu(B(y ; r)) \leqslant c\left(1+\frac{d(x, y)}{r}\right)^{N} \mu(B(x ; r))
$$

uniformly for all $x, y \in \mathcal{X}$ and $r>0$. Indeed, the property (2.2) with $N=n$ is a direct consequence of triangle inequality of the metric $d$ and the strong homogeneity property. In the case of Euclidean spaces $\mathbb{R}^{n}$ and Lie groups of polynomial growth, $N$ can be chosen to be 0 .

The standard Hardy-Littlewood maximal function $M_{r} f, 1 \leqslant r<\infty$ is defined by

$$
M_{r} f(x)=\sup _{x \in B}\left(\frac{1}{\mu(B)} \int_{B}|f(y)|^{r} d \mu(y)\right)^{1 / r},
$$


where the sup is taken over all balls containing $x$. If $r=1, M_{1} f$ will be denoted by $M f$. The Fefferman-Stein sharp maximal function of $f, f^{\#}(x)$, is defined by

$$
f^{\#}(x)=\sup _{B \ni x} \frac{1}{\mu(B)} \int_{B}\left|f(y)-f_{B}\right| d \mu(y),
$$

where $f_{B}=1 /(\mu(B)) \int_{B} f d \mu$. We will say $f \in B M O(\mathcal{X})$ if $f \in L_{\text {loc }}^{1}(\mathcal{X})$ and $f^{\#}(x) \in L^{\infty}$. If $f \in B M O$, the $B M O$ semi-norm of $f$ is given by

$$
\|f\|_{*}=\sup _{\boldsymbol{x}} f^{\#}(x)=\sup _{x} \sup _{B \ni x} \frac{1}{\mu(B)} \int_{B}\left|f(y)-f_{B}\right| d \mu(y) .
$$

A family of operators $A_{t}, t>0$ is said to be "generalised approximations to the identity" if, for every $t>0, A_{t}$ can be represented by kernels $a_{t}(x, y)$ in the following sense: for every function $f \in L^{p}(\mathcal{X}), p \geqslant 1, A_{t} f(x)=\int_{\mathcal{X}} a_{t}(x, y) f(y) d \mu(y)$, and the following condition holds:

$$
\left|a_{t}(x, y)\right| \leqslant h_{t}(x, y)=\left(\mu\left(B\left(x ; t^{1 / m}\right)\right)\right)^{-1} s\left(d(x, y)^{m} t^{-1}\right),
$$

in which $m$ is a positive constant and $s$ is a positive, bounded, decreasing function satisfying

$$
\lim _{r \rightarrow \infty} r^{n+N+\epsilon} s\left(r^{m}\right)=0
$$

for some $\epsilon>0$, where $n$ and $N$ are two constants in (2.1) and (2.2).

Note that (2.2) and (2.4) imply that

$$
\left|a_{t}(x, y)\right| \leqslant\left(\mu\left(B\left(y ; t^{1 / m}\right)\right)\right)^{-1} \times\left(1+\frac{d(x, y)}{t^{1 / m}}\right)^{-(n+\epsilon)} .
$$

In [11], the sharp maximal function $M_{A}^{\#} f$ associated with "generalised approximations to the identity" $\left\{A_{t}, t>0\right\}$ is defined by

$$
M_{A}^{\#} f(x)=\sup _{x \in B} \frac{1}{\mu(B)} \int_{B}\left|f(y)-A_{t_{B}} f(y)\right| d \mu(y)
$$

where $t_{B}=r_{B}^{m}$, and $f \in L^{p}(\mathcal{X})$ for some $p \geqslant 1$.

We recall some results, which have been proved in the context of spaces of homogeneous type in $[3,4,8] 2$.

LEMMA 2.1 .

(i) For every $p \in[1, \infty]$, there exists a constant $C$ such that for every $f$ $\in L^{p}(\mathcal{X})$,

$$
A_{t} f(x) \leqslant C M f(x)
$$


(ii) Assume $b \in B M O$ and $M>1$. Then for every ball $B(x ; r)$, we have

$$
\left|b_{B}-b_{M B}\right| \leqslant C\|b\|_{*} \log M ;
$$

(iii) (John-Nirenberg Lemma) Let $1 \leqslant p<\infty$ and $B \subset \mathcal{X}$, then $b \in B M O$ if and only if

$$
\frac{1}{\mu(B)} \int_{B}\left|b-b_{B}\right|^{p} d \mu(x) \leqslant\|b\|_{*}^{p} .
$$

LEMMA 2.2. Let $\lambda>0$ and $f \in L^{p}(\mathcal{X})$ for some $1<p<\infty$. Then for every $0<\eta<1$, we can find $\gamma>0$ independent of $\lambda, f$ in such a way that

$$
\mu\left\{x \in \mathcal{X}: M f(x)>D \lambda, M_{A}^{\#} f(x) \leqslant \gamma \lambda\right\} \leqslant \eta \mu\{x \in \mathcal{X}: M f(x)>\lambda\},
$$

where $D>1$ is a fixed constant which depends only on the space $\mathcal{X}$ and the "generalised approximations of the identity" $\left\{A_{t}, t>0\right\}$.

As a consequence, we have the following estimate:

$$
\|f\|_{p} \leqslant\|M(f)\|_{p} \leqslant C\left\|M_{A}^{\#} f\right\|_{p}
$$

for every $f \in L^{p}(\mathcal{X}), 1<p<\infty$.

For the proof of this lemma, see [11, Proposition 4.1].

LEMMA 2.3. Let $\left\{A_{t}, t>0\right\}$ be a "generalised approximations of the identity" and let $b \in B M O$. Then, for every function $f \in L^{p}(\mathcal{X}), p>1, x \in \mathcal{X}$ and $1<r<\infty$ we have

$$
\sup _{x \in B} \frac{1}{\mu(B)} \int_{B}\left|A_{t_{B}}\left(b-b_{B}\right) f(y)\right| d \mu(y) \leqslant C\|b\|_{*} M_{\tau} f(x),
$$

where $t_{B}=r_{B}^{m}$.

Proof: We fix $f \in L^{p}(\mathcal{X}), p>1 ; x \in \mathcal{X}$ and $x \in B$ for some ball $B$. Then,

$$
\begin{aligned}
\frac{1}{\mu(B)} \int_{B}\left|A_{t_{B}}\left(\left(b-b_{B}\right) f\right)(y)\right| d \mu(y) & \\
\leqslant & \frac{1}{\mu(B)} \int_{B} \int_{\mathcal{X}} h_{t_{B}}(x, y)\left|\left(b(y)-b_{B}\right) f(y)\right| d \mu(y) d \mu(x) \\
& \leqslant \frac{1}{\mu(B)} \int_{B} \int_{2 B} h_{t_{B}}(x, y)\left|\left(b(y)-b_{B}\right) f(y)\right| d \mu(y) d \mu(x) \\
& \quad+\sum_{k=1}^{\infty} \frac{1}{\mu(B)} \int_{B} \int_{2^{k+1} B \backslash 2^{k} B} h_{t_{B}}(x, y)\left|\left(b(y)-b_{B}\right) f(y)\right| d \mu(y) d \mu(x) \\
= & \mathrm{I}+\mathrm{II} .
\end{aligned}
$$

We now estimate I. By (2.2) we have $\mu(B) \leqslant 2^{N} \mu\left(B\left(x, r_{B}\right)\right)$ since $x \in B$. Besides, for $y \in 2 B$ we have

$$
h_{t_{B}}(x, y)=\frac{s\left(d(x, y)^{m} t_{B}^{-1}\right)}{\mu\left(B\left(x ; t_{B}^{1 / m}\right)\right)} \leqslant \frac{s(0)}{\mu\left(B\left(x ; r_{B}\right)\right)} \leqslant \frac{C}{\mu(B)} \leqslant \frac{C}{\mu(2 B)} .
$$


Note that the first inequality is a consequence of the assumption that $s$ is decreasing. Denote $1 / r+1 / r^{\prime}=1$. By using (iii) of Lemma 2.1, we obtain

$$
\begin{aligned}
I & \leqslant C \frac{1}{\mu(B) \mu(2 B)} \int_{B} \int_{2 B}\left|\left(b(y)-b_{B}\right) f(y)\right| d \mu(y) d \mu(x) \\
& \leqslant C \frac{1}{\mu(2 B)} \int_{2 B}\left|\left(b(y)-b_{B}\right) f(y)\right| d \mu(y) \\
& \leqslant C\left[\frac{1}{\mu(2 B)} \int_{2 B}\left|\left(b(y)-b_{B}\right)\right|^{r^{\prime}} d \mu(y)\right]^{1 / r^{\prime}}\left[\frac{1}{\mu(2 B)} \int_{2 B}|f(y)|^{r} d \mu(y)\right]^{1 / r} \\
& \leqslant C\|b\|_{*} M_{r} f(x) .
\end{aligned}
$$

Regarding II, for $x \in B$ and $y \in 2^{k+1} B \backslash 2^{k} B$ we have $d(x, y) \geqslant 2^{k-1} r_{B}$ and from the doubling volume property

$$
h_{t_{B}}(x, y)=\frac{s\left(d(x, y)^{m} t_{B}^{-m}\right)}{\mu\left(B\left(x ; t_{B}^{1 / m}\right)\right)} \leqslant \frac{s\left(2^{(k-1) m}\right)}{\mu(B)} \leqslant \frac{s\left(2^{(k-1) m}\right) 2^{(k+1) n}}{\mu\left(2^{k+1} B\right)} .
$$

By (2.4) and Lemma 2.1, we thus obtain

$$
\begin{aligned}
\mathrm{II} & \leqslant C \sum_{k=1}^{\infty} 2^{k n} s\left(2^{(k-1) m}\right) \frac{1}{\mu(B) \mu\left(2^{k+1} B\right)} \int_{B} \int_{2^{k+1} B}\left|\left(b(y)-b_{B}\right) f(y)\right| d \mu(y) d \mu(x) \\
& \leqslant C \sum_{k=1}^{\infty} 2^{k n} s\left(2^{(k-1) m}\right) \frac{1}{\mu\left(2^{k+1} B\right)} \int_{2^{k+1} B}\left|\left(b(y)-b_{B}\right) f(y)\right| d \mu(y) \\
& \leqslant C\|b\|_{*} \sum_{k=1}^{\infty}(k+1) 2^{k n} s\left(2^{(k-1) m}\right) M_{r} f(x) \\
& \leqslant C\|b\|_{*} M_{\tau} f(x) .
\end{aligned}
$$

The proof of Lemma 2.3 is complete.

\section{The MAIN THEOREM AND ITS PROOF}

Assume that $\mathcal{X}$ is a space of homogeneous type of infinite measure, equipped with a metric $d$ and a measure $\mu$. In this section, we assume the following:

(a) $T$ is a bounded operator on $L^{2}(\mathcal{X})$.

(b) There exists an "generalised approximations of the identity" $\left\{B_{t}, t>0\right\}$ such that $\left(T-T B_{t}\right)$ have associated kernels $K_{t}(x, y)$ and there exist positive constants $c_{1}, c_{2}$ such that

$$
\int_{d(x, y)>c_{1} t^{1 / m}}\left|K_{t}(x, y)\right| d \mu(x) \leqslant c_{2}, \quad \text { for all } y \in \mathcal{X} .
$$

(c) There exists an "generalised approximations of the identity" $\left\{A_{t}, t>0\right\}$ such that the kernels $k_{t}(x, y)$ of the operators $\left(T-A_{t} T\right)$ satisfy 


$$
\left|k_{t}(x, y)\right| \leqslant c_{4} \frac{1}{\mu(B(x ; d(x, y))} \frac{t^{\alpha / m}}{d(x, y)^{\alpha}},
$$

then $d(x, y) \geqslant c_{3} t^{1 / m}$ for some. $c_{3}, c_{4}, \alpha>0$.

It is proved in [8] that if $T$ is an operator satisfying (a) and (b) above, then $T$ is of weak type $(1,1)$ and of strong type $(p, p)$ for $1<p \leqslant 2$. In addition, if (c) is also satisfied, the operator $T$ is bounded on $L^{p}(\mathcal{X})$ for all $1<p<\infty$.

The aim of this section is to prove the following theorem.

THEOREM 3.1. Let $T$ be an operator satisfying the above conditions (a), (b) and (c) and $b \in B M O$. Then the commutator $[b, T]$ can be extended to $L^{p}(\mathcal{X})$ continuously, and there exists a constant $C=C(p, T, \mathcal{X})$ such that

$$
\|[b, T] f\|_{p} \leqslant C\|b\|_{*}\|f\|_{p}
$$

for $1<p<\infty$.

Proof: Choose two real numbers $r$ and $s$ greater than 1 such that $1<r s<p<\infty$. We will prove that there exists a constant $C=C(p, T, \mathcal{X})$ such that

$$
M_{A}^{\#}([b, T])(f)(x) \leqslant C\|b\|_{*} M_{r} T f(x)+C\|b\|_{*} M_{r s} f(x)
$$

for all $x \in \mathcal{X}$.

From (3.3) and (2.6), (3.2) follows by the continuity of $T$, and of the maximal inequality ([13]),

$$
\|[b, T] f\|_{p} \leqslant C\left\|M_{A}^{\#}([b, T]) f\right\|_{p} \leqslant C\|b\|_{*}\left(\left\|M_{r} T f\right\|_{p}+\left\|M_{r s} f\right\|_{p}\right) \leqslant C\|b\|_{*}\|f\|_{p}
$$

for all $1<r s<p<\infty$.

We now prove (3.3). For an arbitrary fixed $x \in \mathcal{X}$, choose a ball $B\left(x_{0} ; r\right)=\{y$ $\left.\in \mathcal{X}: d\left(x_{0}, y\right)<r\right\}$ which contains $x$. Let $f_{1}=f \chi_{2 B}$ and $f_{2}=f-f_{1}$. One writes

$$
[b, T] f=\left(b-b_{B}\right) T f-T\left(\left(b-b_{B}\right) f_{1}\right)-T\left(\left(b-b_{B}\right) f_{2}\right),
$$

and

$$
A_{t_{B}}([b, T] f)=A_{t_{B}}\left(\left(b-b_{B}\right) T f\right)-A_{t_{B}} T\left(\left(b-b_{B}\right) f_{1}\right)-A_{t_{B}} T\left(\left(b-b_{B}\right) f_{2}\right),
$$

where $t_{B}=r_{B}^{m}$. Then, by $(2.5)$

$$
\begin{aligned}
& \frac{1}{\mu(B)} \int_{B}\left|[b, T] f(y)-A_{t_{B}}[b, T] f(y)\right| d \mu(y) \\
& \quad \leqslant \frac{1}{\mu(B)} \int_{B}\left|\left(b(y)-b_{B}\right) T f(y)\right| d \mu(y)+\frac{1}{\mu(B)} \int_{B}\left|T\left(\left(b(y)-b_{B}\right) f_{1}\right)(y)\right| d \mu(y)
\end{aligned}
$$




$$
\begin{aligned}
& +\frac{1}{\mu(B)} \int_{B}\left|A_{t_{B}}\left(\left(b(y)-b_{B}\right) T f\right)(y)\right| d \mu(y) \\
& +\frac{1}{\mu(B)} \int_{B}\left|A_{t_{B}} T\left(\left(b(y)-b_{B}\right) f_{1}\right)(y)\right| d \mu(y) \\
& +\frac{1}{\mu(B)} \int_{B}\left|T\left(\left(b-b_{B}\right) f_{2}\right)(y)-A_{t_{B}} T\left(\left(b-b_{B}\right) f_{2}\right)(y)\right| d \mu(y) \\
& =\mathrm{I}+\mathrm{II}+\mathrm{III}+\mathrm{IV}+\mathrm{V} .
\end{aligned}
$$

Let $r^{\prime}$ be the dual of $r$ such that $1 / r+1 / r^{\prime}=1$. Using the Hölder inequality and Lemma 2.1 , we have

$$
\begin{aligned}
I & \leqslant\left[\frac{1}{\mu(B)} \int_{B}\left|b(y)-b_{B}\right|^{r^{\prime}} d \mu(y)\right]^{1 / r^{\prime}}\left[\frac{1}{\mu(B)} \int_{B}|T f(y)|^{r} d \mu(y)\right]^{1 / r} \\
& \leqslant C\|b\|_{*} M_{r} T f(x) .
\end{aligned}
$$

By Lemma 2.1 and the $L^{p}$ boundedness of $T$,

$$
\begin{aligned}
\mathrm{II} & \left.\leqslant\left.\left(\frac{1}{\mu(B)} \int_{\mathcal{X}} \mid T\left(\left(b(y)-b_{B}\right)\right) f_{1}\right)(y)\right|^{s} d \mu(y)\right)^{1 / s} \\
& \leqslant C\left(\frac{1}{\mu(B)} \int_{2 B}\left|\left(b(y)-b_{B}\right) f(y)\right|^{s} d \mu(y)\right)^{1 / s} \\
& \leqslant C\left[\frac{1}{\mu(B)} \int_{2 B}\left|b(y)-b_{B}\right|^{s r^{\prime}} d \mu(y)\right]^{1 / s r^{\prime}}\left[\frac{1}{\mu(B)} \int_{2 B}|f(y)|^{s r} d \mu(y)\right]^{1 / s r} \\
& \leqslant C\|b\|_{*} M_{r s} f(x) .
\end{aligned}
$$

Similarly, by Lemmas $2.1,2.3$ and the $L^{p}$ boundedness of $T$, we obtain

$$
\mathrm{III}+\mathrm{IV} \leqslant C\|b\|_{*} M_{r} T f(x)+C\|b\|_{*} M_{r s} f(x) .
$$

We now consider the term V. Using the assumption (c), we have

$$
\begin{aligned}
\mathrm{V} \leqslant & \frac{1}{\mu(B)} \int_{B} \int_{(2 B)^{c}}\left|k_{t_{B}}(y, z)\right|\left|\left(b(z)-b_{B}\right) f(z)\right| d \mu(z) d \mu(y) \\
\leqslant & C \sum_{k=1}^{\infty} \int_{2^{k} r_{B} \leqslant d\left(x_{0}, z\right)<2^{k+1} r_{B}} \frac{1}{\mu\left(B\left(x_{0} ; d\left(x_{0}, z\right)\right)\right)} \frac{r_{B}^{\alpha}}{d\left(x_{0}, z\right)^{\alpha}}\left|\left(b(z)-b_{B}\right) f(z)\right| d \mu(z) \\
\leqslant & C \sum_{k=1}^{\infty} 2^{-k \alpha} \frac{1}{\mu\left(B\left(x_{0} ; 2^{k} r_{B}\right)\right)} \int_{d\left(x_{0}, z\right)<2^{k+1} r_{B}}\left|\left(b(z)-b_{B}\right) f(z)\right| d \mu(z) \\
\leqslant & C \sum_{k=1}^{\infty} 2^{-k \alpha} \frac{1}{\mu\left(B\left(x_{0} ; 2^{k} r_{B}\right)\right)} \int_{d\left(x_{0}, z\right)<2^{k+1} r_{B}}\left|b(z)-b_{2^{k+1} B}\right||f(z)| d \mu(z) \\
& +C \sum_{k=1}^{\infty} 2^{-k \alpha}\left|b_{2^{k+1} B}-b_{B}\right| \frac{1}{\mu\left(B\left(x_{0} ; 2^{k} r_{B}\right)\right)} \int_{d\left(x_{0}, z\right)<2^{k+1} r_{B}}|f(z)| d \mu(z) \\
\leqslant & C\|b\|_{*} \sum_{k=1}^{\infty} 2^{-k \alpha} M_{r} f(x)+C\|b\|_{*} \sum_{k=1}^{\infty} 2^{-k \alpha}(k+1) M f(x)
\end{aligned}
$$




$$
\leqslant C\|b\|_{*} M_{r} f(x)
$$

Combining the above estimates of I, II, III, IV and V, we obtained (3.3). The proof of Theorem 3.1 is complete.

\section{ThE CASE OF MEASURABLE SUBSETS OF SPACES OF HOMOgENEOUS TYPE}

We now assume that $\Omega$ is a measurable subset of the space of homogeneous type $(\mathcal{X}, d, \mu)$. An example of $\Omega$ is an open domain of the Euclidean space $\mathbb{R}^{n}$. If $\Omega$ possesses certain smoothness on its boundary, for example Lipschitz boundary, then it is a space of homogeneous type and Theorem 3.1 is applicable. However, a general measurable set $\Omega$ needs not satisfy the doubling property, hence it is not a space of homogeneous type. Such a measurable set $\Omega$ appears naturally in boundary value problems, for example for partial differential equations with Dirichlet boundary conditions.

We are interested in dealing with the commutator estimates in these context. Following the approach in [8], we extend the singular integrals defined on $\Omega$ to the space $\mathcal{X}$. Since there is no assumption on the regularity of the kernels in the space variables, the extension of the kernel still satisfy similar conditions. Given a linear operator $T$ which maps $L^{p}(\Omega)$ into itself for some $p$, defined an associated operator $\widetilde{T}$ on $L^{p}(\Omega)$ by

$$
\tilde{T}(f)(x)=T\left(f \chi_{\Omega}\right)(x) \chi_{\Omega}(x),
$$

where $\chi_{\Omega}$ is the characterisatic function on $\Omega$. Then $T$ is bounded on $L^{p}(\Omega)$ if and only if $\widetilde{T}$ is bounded on $L^{p}(\mathcal{X})$. Note that if $T$ has an associated kernel $K(x, y)$ in the sense of (1.1), then $\widetilde{T}$ also has an associated kernel $\widetilde{K}(x, y)$ in the sense of (1.1), given by

$$
\widetilde{K}(x, y)=K(x, y) \chi_{\Omega \times \Omega}(x, y) .
$$

It is easy to see that the pointwise Hörmander condition for $K$ would not necessarily imply such a condition for $\widetilde{K}$ because this kernel can be discontinuous. However, the kernel assumptions (b) and (c) do not involve their regularity, and they induce similar properties on the kernels of the extended operators.

From now on, to differentiate between a ball in $\mathcal{X}$ and a ball in $\Omega$, we use the notations $B^{\mathcal{X}}$ and $B^{\Omega}$. A "generalised approximations to the identity" $\left\{A_{t}, t>0\right\}$ in this context is given by

$$
A_{t} f(x)=\int_{\mathcal{X}} a_{t}(x, y) f(y) d \mu(y), \quad f \in L^{p}(\Omega), \text { for some } p \geqslant 1,
$$

where the kernels $a_{t}(x, y)$ satisfy the following conditions:

$$
\left|a_{t}(x, y)\right| \leqslant h_{t}(x, y)
$$


for all $x, y \in \Omega$, where $h_{t}(x, y)$ is defined on $\mathcal{X} \times \mathcal{X}$ by

$$
h_{t}(x, y)=\left(\mu\left(B^{\mathcal{X}}\left(x ; t^{1 / m}\right)\right)\right)^{-1} s\left(d(x, y)^{m} t^{-1}\right)
$$

in which $m$ is a positive constant and $s$ is a positive, bounded, decreasing function satisfying (2.4). We assume the following:

(a') $T$ is a bounded operator on $L^{2}(\Omega)$ with kernel $K(x, y)$, such that, for every $f \in L_{0}^{\infty}(\Omega)$,

$$
T(f)(x)=\int_{\mathcal{X}} K(x, y) f(y) d \mu(y) \text { for } \mu-\text { almost all } x \notin \operatorname{supp} f .
$$

$\left(b^{\prime}\right)$ There exists a "generalised approximations of the identity" $\left\{B_{t}, t>0\right\}$ such that $\left(T-T B_{t}\right)$ have associated kernels $K_{t}(x, y)$ and there exist positive constants $c_{1}, c_{2}$ so that

$$
\int_{d(x, y)>c_{1} t^{1 / m}}\left|K_{t}(x, y)\right| d \mu(x) \leqslant c_{2}, \quad \text { for all } y \in \Omega .
$$

(c') There exists a "generalised approximations of the identity" $\left\{A_{t}, t>0\right\}$ such that the kernels $k_{t}(x, y)$ of the operators $\left(T-A_{t} T\right)$ satisfy

$$
\left|k_{t}(x, y)\right| \leqslant c_{4} \frac{1}{\mu(B(x ; d(x, y))} \frac{t^{\alpha / m}}{d(x, y)^{\alpha}},
$$

when every $x, y \in \Omega$ with $d(x, y) \geqslant c_{3} t^{1 / m}$ for some $c_{3}, c_{4}, \alpha>0$,

In [8] it is proved that if $T$ satisfies (a') and (b'), then it is of weak type $(1,1)$ and hence bounded on $L^{p}(\Omega)$ for $1<p \leqslant 2$. The proof of these estimates is based on extending the operators to $\mathcal{X}$, then showing that conditions $\left(\mathrm{a}^{\prime}\right),\left(\mathrm{b}^{\prime}\right)$ and $\left(\mathrm{c}^{\prime}\right)$ on $T, A_{t}$, $B_{t}$ imply that $\tilde{T}$ satisfies (a), (b) and (c) on $\mathcal{X}$. Hence, the previous results on spaces of homogeneous type $\mathcal{X}$ can be applied to obtain the following theorem.

TheOREM 4.1. Assume that $T$ is an operator satisfying the above conditions $\left(a^{\prime}\right),\left(b^{\prime}\right)$ and $\left(c^{\prime}\right)$. Let $b(x)$ be defined on $\Omega$ such that $\tilde{b}(x)$ is in $B M O(\mathcal{X})$ where $\tilde{b}(x)$ $=b(x) \chi_{\Omega}(x)$. Then, for every $f \in L^{p}(\Omega)$, there exists a constant $C=C(p, T, \Omega)$ such that

$$
\|[b, T] f\|_{L^{\mathcal{P}}(\Omega)} \leqslant C\|\widetilde{b}\|_{*}\|f\|_{L^{p}(\Omega)}
$$

for all $1<p<\infty$.

\section{Holomorphic FUNCTIONAL CALCULI OF LINEAR ELLIPTIC OPERATORS}

We first review some definitions regarding the holomorphic functional calculus as introduced by McIntosh [12]; see also [1]. Let $0 \leqslant \omega<\pi$ be given. Then

$$
S_{\omega}=\{z \in \mathbb{C}:|\arg z| \leqslant \omega\} \cup\{0\}
$$


denotes the closed sector of angle $\omega$ and $S_{\omega}^{0}$ denotes its interior, while $\dot{S}_{\omega}=S_{\omega} \backslash\{0\}$. An operator $L$ on some Banach space $E$ is said to be of type $\omega$ if $L$ is closed and densely defined, $\sigma(L) \subset S_{\omega}$, and for each $\theta \in(\omega, \pi]$ there exists a constant $C_{\theta}$ such that

$$
|\eta|\left\|(\eta I-L)^{-1}\right\|_{\mathcal{L}(E)} \leqslant C_{\theta}, \quad \eta \in-\dot{S}_{\pi-\theta} .
$$

If $\mu \in(0, \pi]$, then

$$
H_{\infty}\left(S_{\mu}^{0}\right)=\left\{f: S_{\mu}^{0} \rightarrow \mathbb{C} ; f \text { is holomorphic and }\|f\|_{\infty}<\infty\right\},
$$

where $\|f\|_{\infty}=\sup \left\{|f(z)|: z \in S_{\mu}^{0}\right\}$. In addition, we define

$$
\Psi\left(S_{\mu}^{0}\right)=\left\{g \in H_{\infty}\left(S_{\mu}^{0}\right): \exists s>0, \exists c \geqslant 0:|g(z)| \leqslant c \frac{|z|^{s}}{1+|z|^{2 s}}\right\} .
$$

If $L$ is of type $\omega$ and $g \in \Psi\left(S_{\mu}^{0}\right)$, we define $g(L) \in L(E)$ by

$$
g(L)=-\frac{1}{2 \pi i} \int_{\Gamma}(\eta I-L)^{-1} g(\eta) d \eta
$$

where $\Gamma$ is the contour $\left\{\xi=r e^{ \pm i \theta}: r \geqslant 0\right\}$ parametrised clockwise around $S_{\omega}$, and $\omega<\theta<\mu$. If, in addition, $L$ is one-one and has dense range and if $f \in H_{\infty}\left(S_{\mu}^{0}\right)$, then

$$
f(L)=[h(L)]^{-1}(f h)(L),
$$

where $h(z)=z(1+z)^{-2}$. It can be shown that $f(L)$ is a well-defined linear operator in $E$ and that this definition is consistent with definition (5.2) for $f \in \Psi\left(S_{\mu}^{0}\right)$. The definition of $f(L)$ can even extended to encompass unbounded holomorphic functions; see [12] for details. $L$ is said to have a bounded holomorphic functional calculus on the sector $S_{\mu}$ if

$$
\|g(L)\|_{\mathcal{L}(E)} \leqslant N\|g\|_{\infty}
$$

for some $N>0$, and for all $g \in H_{\infty}\left(S_{\mu}^{0}\right)$.

Coming back to our setting, let us suppose that $\Omega$ is a measurable subset of a space of homogeneous type $\mathcal{X}$. Let $L$ be a linear operator on $L^{2}(\Omega)$ with $\omega<\pi / 2$ so that $(-L)$ generates a holomorphic semigroup $e^{-z L}, 0 \leqslant|\operatorname{Arg}(z)|<\pi / 2-\omega$. Applying [8, Theorem $6]$ and Theorem 4.1, we have

THEOREM 5.1. Assume the following three conditions.

(a) The holomorphic semigroup $e^{-z L},|\operatorname{Arg}(z)|<\pi / 2-\omega$ is represented by kernels $a_{z}(x, y)$ which satisfy, for all $\theta>\omega$, an upper bound

$$
\left|a_{z}(x, y)\right| \leqslant c_{\theta} h_{|z|}(x, y)
$$

for $x, y \in \Omega$, and $|\operatorname{Arg}(z)|<\pi / 2-\theta$, where $h_{|z|}$ is defined on $\mathcal{X} \times \mathcal{X}$ by (2.5). 
(b) The operator $L$ has a bounded holomorphic functional calculus in $L^{2}(\Omega)$. That is, for any $\nu>\omega$ and $g \in H_{\infty}\left(S_{\nu}^{0}\right)$, the operator $g(L)$ satisfies

$$
\|g(L) f\|_{L^{2}(\Omega)} \leqslant c_{\nu}\|g\|_{\infty}\|f\|_{L^{2}(\Omega)} .
$$

(c) Let $b(x)$ be defined on $\Omega$ such that $\tilde{b}(x)$ is in $B M O(\mathcal{X})$ where $\tilde{b}(x)$ $=b(x) \chi_{\Omega}(x)$.

Then, for every $1<p<\infty$, the commutator $[b, g(L)]$ is bounded on $L^{p}(\Omega)$, that is

$$
\|[b, g(L)] f\|_{L^{p}(\Omega)} \leqslant C\|\widetilde{b}\|_{*}\|g\|_{\infty}\|f\|_{L^{p}(\Omega)}
$$

for all $g \in H_{\infty}\left(S_{\nu}^{0}\right)$.

NotE. Using assumption (a) and (5.1), it was proved in ([8, Theorem 6]) that the conditions $\left(b^{\prime}\right)$ and $\left(c^{\prime}\right)$ of Theorem 4.1 are satisfied.

Applications. Theorem 5.1 gives new results when we do not assume smoothness of heat kernels in the space variables, or when $\Omega$ is a measurable set with no smoothness on its boundary.

(a) Let $V$ be a nonnegative function on $\mathbb{R}^{n}$. The Schrödinger operator with potential $V$ is defined by

$$
L=-\Delta+V(x)
$$

The Trotter formula shows that the semigroup $e^{-t L}$ has a kernel $p_{t}(x, y)$ which satisfies an upper bound of Gaussian type, that is

$$
0<p_{t}(x, y) \leqslant \frac{C}{t^{n / 2}} e^{-c\left(|x-y|^{2} / t\right)}
$$

for $x, y \in \mathbb{R}^{n}$ and all $t>0$. However, unless $V$ satisfies additional conditions, the heat kernel can be a discontinuous function of the space variables and the Hölder continuous estimates may fail to hold. See, $[\mathbf{1}$, Lecture 7$]$.

Applying Theorem 5.1 , the commutator $[b, g(L)]$ is bounded on $L^{p}\left(\mathbb{R}^{n}\right)$ for all 1 $<p<\infty$, that is,

$$
\|[b, g(L)] f\|_{L^{p}\left(\mathbb{R}^{n}\right)} \leqslant C\|b\|_{*}\|g\|_{\infty}\|f\|_{L^{p}\left(\mathbb{R}^{n}\right)}
$$

for all $g \in H_{\infty}\left(S_{\nu}^{0}\right)$.

(b) Let

$$
L f=-\sum_{i, j=1}^{n} \frac{\partial}{\partial x_{i}} a_{i j}(x) \frac{\partial}{\partial x_{j}} f
$$

be the elliptic divergence form operator of real, symmetric coefficients with Dirichlet boundary conditions on a domain $\Omega$ of $\mathbb{R}^{n}$ which is defined by the variational method. This means that $L$ is the positive self-adjoint operator associated with the form

$$
Q(f, g)=\int_{\Omega} \sum_{i, j=1}^{n} a_{i j}(x) \frac{\partial}{\partial x_{j}} f \cdot \frac{\partial}{\partial x_{i}} \bar{g}
$$


on $V \times V$ by $\langle L f, g\rangle=Q(f, g)$, where $V$ is the Sobolev space $H_{0}^{1}(\Omega)$. It is known that the operator $L$ has Gaussian heat kernel bounds without any conditions on smoothness of the boundary of $\Omega$. See [7]. Therefore, when $\widetilde{b}(x)=b(x) \chi_{\Omega}(x)$ is in $B M O\left(\mathbb{R}^{n}\right)$, Theorem 5.1 shows that for all $g \in H_{\infty}\left(S_{\nu}^{0}\right)$, the commutator $[b, g(L)]$ is a bounded operator on $L^{p}(\Omega)$.

More general operators on open domain of $\mathbb{R}^{n}$ which possess Gaussian bounds can be found in $[1,2,8]$.

\section{REFERENCES}

[1] D. Albrecht, X.T. Duong and A. McIntosh, 'Operator theory and harmonic analysis', in Instructional Workshop on Analysis and Geometry, Proc. Centre Math. Analysis 34 (A.N.U., Canberra, 1996), pp. 77-136.

[2] W. Arendt and A.F.M. ter Elst, 'Gaussian estimates for second order elliptic operators with boundary conditions', J. Operator Theory 38 (1997), 87-130.

[3] M. Bramanti and M. Cerutti, 'Commutators of singular integrals on homogeneous spaces', Boll. Un. Mat. Ital. 10 (1996), 843-883.

[4] N. Burger, 'Espace des fonctions variation moyenne borne sur un espace de nature homogène', C. R. Acad. Sci. Paris Sr. 286 (1978), 139-142.

[5] R.R. Coifman, R. Rochberg and G. Weiss, 'Factorization theorem for Hardy spaces in several variables', Ann. of Math. 103 (1976), 611-635.

[6] R.R. Coifman and G. Weiss, Analyse harmonique non-commutative sur certains espaces homognès, Lecture Notes in Math. 242 (Springer-Verlag, Berlin, Heidelberg, New York, 1971).

[7] E.B. Davies, Heat kernels and spectral theory (Cambridge Univ. Press, Cambridge, 1989).

[8] X.T. Duong and A. McIntosh, 'Singular integral operators with non-smooth kernels on irregular domains', Rev. Mat. Iberoamericana 15 (1999), 233-265.

[9] X.T. Duong and L.X. Yan, 'Commutators of BMO functions and singular integral operators with non-smooth kernels on spaces of finite measure', (in preparation).

[10] S. Janson, 'Mean oscillation and commutators of singular integrals operators', Ark. Mat. 16 (1978), 263-270.

[11] J.M. Martell, 'Sharp maximal functions associated with approximations of the identity in spaces of homogeneous type and applications', (preprint, 2001).

[12] A. McIntosh, 'Operators which have an $H_{\infty}$-calculus', in Miniconference on Operator Theory and Partial Differential Equations, Proc. Centre Math. Analysis 14 (A.N.U., Canberra, 1986), pp. 210-231.

[13] E.M. Stein, Harmonic analysis: Real variable methods, orthogonality and oscillatory integrals, Princeton Mathematical Seriex 43 (Princeton Univ. Press, Princeton, N.J., 1993). 
Department of Mathematics

Macquarie University

New South Wales 2109

Australia

e-mail: duong@ics.mq.edu.au
Department of Mathematics

Macquarie University

New South Wales 2109

Australia

e-mail: lixin@ics.mq.edu.au

and

Department of Mathematics

Zhongshan University Guangzhou 510275

Peoples Republic of China 